\section{Enseñanza, investigación y extensión: tres prácticas articuladas en la cátedra de Didáctica General de la Universidad Nacional del Litoral}

\section{Victoria Baraldi}

Docente investigadora de la Facultad

de Humanidades y Ciencias.

Universidad Nacional del Litoral,

Argentina.

vbaraldi@fhuc.unl.edu.ar
Integración de la docencia y la extensión /

Intervenciones

RECEPCIÓN: 24/06/16

ACEPTACIÓN FINAL: 10/10/16

\section{Resumen}

En el presente artículo se expresan los modos en que al interior de una cátedra se vinculan y retroalimentan la práctica de extensión con las prácticas de enseñanza y de investigación.

En primer lugar, se presentan las dos principales coordenadas desde donde se escribe el texto: La Universidad Nacional del Litoral y el campo de la didáctica. Allí se mencionan rasgos identitarios de la creación de esta institución que tienen vigencia en la actualidad y algunas de las particularidades de la didáctica.

En segundo lugar, se describen tres situaciones concretas en las que en la cátedra de Didáctica General se conjugaron las tres funciones sustantivas de la universidad, focalizando en los modos en que una de ellas influyó particularmente sobre las otras. Finalmente, se enuncian los principales principios de la cátedra mencionada.

\section{Resumo}

No presente artigo se expressam os modos que, dentro de uma disciplina, se vinculam e retroalimentam a prática de extensão com as práticas de ensino e de pesquisa.

Em primeiro lugar, apresentam-se as duas principais coordenadas desde onde se escreve o texto: a Universidad Nacional del Litoral e o campo da didática. Aí se nomeiam características identitárias da criação desta instituição, que têm vigência na atualidade e algumas das particularidades da didática.

Em segundo lugar, descrevem-se três situações concretas que, na disciplina Didática Geral, conjugaram-se as três funções substantivas da universidade, focalizando nos modos em que uma delas influenciou particularmente nas outras. Finalmente se enunciam os princípios básicos da disciplina mencionada.
Palabras-clave

- Didáctica

- Enseñanza

- Extensión

- Investigación

- Formación docente
Palavras-chave

- Didática

- Ensino

- Extensão

- Pesquisa

- Formação docente
Para citación de este artículo Baraldi, V. (2016). Enseñanza, investigación y extensión: tres prácticas articuladas en la cátedra de Didáctica General de la Universidad Nacional del Litoral. En Revista +E versión digital, (6), pp. 306-313. Santa Fe, Argentina: Ediciones UNL. 


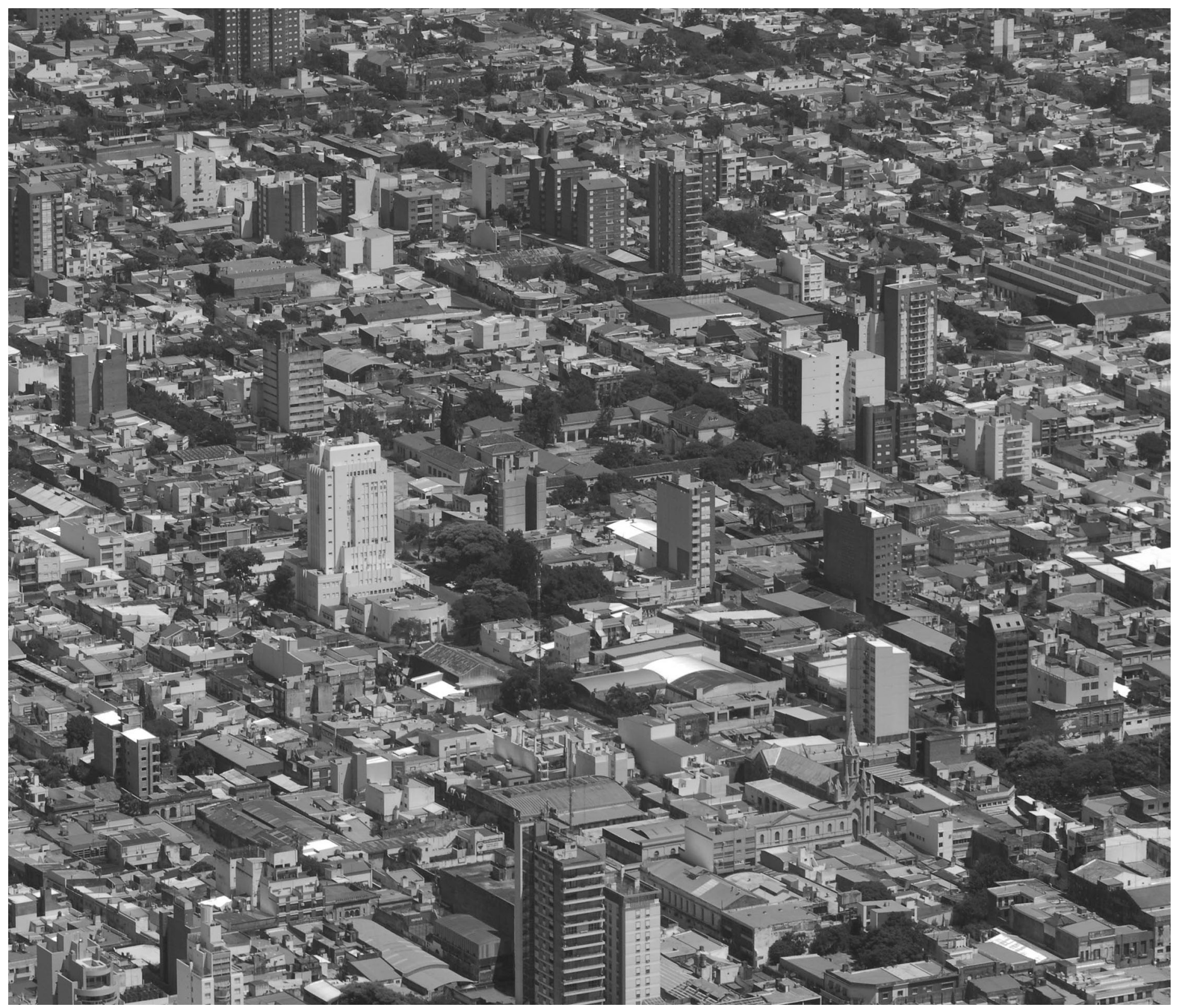

(C) Hugo Pascucci 


\section{Dos coordenadas que encuadran el trabajo}

Los relatos que se incluyen aquí se inscriben en dos coordenadas centrales: una institución: la Universidad Nacional del Litoral (UNL), y un campo de conocimiento: la didáctica. A efectos de una mejor comprensión de lo que describiremos en la segunda parte de este artículo, daremos cuenta primero de ciertos rasgos identitarios de esta institución y explicaremos brevemente el objeto y propósitos de la didáctica.

La UNL fue fundada sobre la base de la Universidad Provincial de Santa Fe creada en 1889. Luego de diversos e intensos debates parlamentarios, la Ley 10861 de 1919 dio origen a la actual UNL. Su creación posee al menos dos características que la distinguen de las creadas con anterioridad en la República Argentina. Por un lado, su conformación se dio a posteriori de la Reforma universitaria, proceso que tuvo un especial protagonismo de los estudiantes, quienes junto a otros actores bregaron, entre otras proclamas, por el cogobierno y la libertad de cátedra. Al decir de muchos, nace "deudora" de la Reforma. Por otro lado, su conformación inicial se asentó sobre la idea de una universidad regional, conformada con siete sedes ubicadas en tres provincias del litoral argentino. ${ }^{1}$ Luego, distintas circunstancias provocaron un desmembramiento que originó tres nuevas universidades. ${ }^{2}$ Pero la impronta de primera universidad regional le dio desde un principio un fuerte vínculo con su medio y permite comprender - sumada a las perspectivas de quienes estuvieron en su conducción en las primeras décadas- la definición de estrategias que hicieron posible que la Universidad no se circunscribiese al otorgamiento de títulos sino que, dentro de la primera década de funcionamiento, tuviese entre sus miras la concreción de las funciones sustantivas de la universidad. En tal sentido, es dable destacar la referencia al Instituto Social, creado en 1927 e inaugurado en 1928, el cual contaba con tres secciones: la Universidad Popular, la Extensión Universitaria y el Museo Social. La primera especialmente destinada a obreros y empleados para un mayor conocimiento de las artes que practicaban, la segunda orientada a la amplia difusión de la cultura intelectual, con el propósito de difundir "el más preciso conocimiento de las necesidades del pueblo y sus condiciones de vida, a fin de provocar el estudio y la solución de los problemas que le atañen" (art. 4); y en el Museo Social se estudiarían "los problemas de índole económico y todos aquellos otros que tengan atingencia directa o indirecta con la evolución y desarrollo del país” (art. 5).
Un estudio del funcionamiento de este Instituto nos permite visualizar que, a pesar de las vicisitudes de las políticas nacionales que siempre repercutieron en la vida universitaria, se fueron conformando desde sus inicios las funciones de investigación y extensión.

Sin ánimo de realizar un recorrido histórico de la UNL, hoy, a casi cien años de su creación, podemos afirmar que se ratifica en su estatuto de 2012 el compromiso con el desarrollo de problemáticas regionales y se enuncia expresamente la necesidad de articular docencia, investigación y extensión Tales propósitos también quedan expresados en el Plan de desarrollo Institucional 2010-2019 y en una de sus líneas de orientación prioritarias. Con estas breves notas queremos enfatizar que la proyección de la Universidad con relación al medio y la necesaria articulación de las tres funciones ha estado presente desde sus primeros años de funcionamiento y forma parte de sus políticas actuales, las cuales se acompañan de instrumentos específicos para hacerlo posible. ${ }^{3}$ La otra coordenada, la didáctica, la cuadriplica en años. Si bien el término didáctica ya había sido utilizado por distintos pedagogos alemanes, existe cierta convención en asociar su "surgimiento" con la escritura de Juan Amós Comenio de su Didáctica Magna en una primera versión en checo concluida en 1632. Este pastor protestante apelaba a la necesidad de educar a la juventud como un modo de superar la crisis por la que estaba atravesando Europa, conmovida por la Guerra de los 30 años. Comenio buscó en la educación, no sólo alcanzar la paz sino la salvación del género humano. Tomando distintas referencias (el pensamiento de grandes filósofos, la observación de la naturaleza y su cosmovisión religiosa) plantea a sus contemporáneos el desafío de "enseñar todo a todos". Cuando dice a todos se refiere a ricos y pobres, nobles y plebeyos, niños y niñas. Cuando se refiere a todo, advierte sobre la imposibilidad y la innecesaridad de hacerlo de un modo exhaustivo, y luego plantea principios para la enseñanza de las ciencias, las artes, los idiomas, las costumbres y la piedad. Hoy, salvando muchísimas distancias, la didáctica guarda para sí el mismo lema, educar a todos, traducido en el cometido de hacer posible la enseñanza como parte de un derecho universal a la educación. Si bien denominamos de otra forma a los destinatarios y son otros los objetos de enseñanza, la educación pública sigue necesitando de un campo de conocimiento específico que permita comprender las particulares relaciones entre quien enseña y quien aprende, mediado por un contenido. Discernir el qué, el por
1) Facultad de Ciencias Jurídicas y Sociales y Facultad de Química Industrial y Agrícola en la ciudad de Santa Fe, Facultad de Ciencias Médicas, Farmacia y Ramos Menores, Facultad de Ciencias Matemáticas,
Físico Química y Naturales Aplicadas a la Industria y Facultad de Ciencias Económicas, Comerciales y Políticas en la ciudad de Rosario, Facultad de Ciencias Económicas y Educacionales en la ciudad de Paraná y Facultad de
Agricultura, Ganadería e Industrias afines con asiento en Corrientes. 2) De la Universidad Nacional del Litoral se conformaron luego la Universidad Nacional del Nordeste, la Universidad Nacional de Rosario y la Universidad
Nacional de Entre Ríos.

3) No redundaremos sobre esta información. Se puede consultar en la página de la UNL los documentos relativos al proceso de autoevaluación institucional. 
qué y para qué, como también el cómo para que la enseñanza sea posible, requiere de un campo de conocimiento que logre comprender esta práctica compleja. De esta manera, "la didáctica es una teoría de la enseñanza, heredera y deudora de muchas otras disciplinas" (Camilloni, 1996:39) que busca no solo comprender sino intervenir en la enseñanza.

En nuestro caso, la didáctica asume una definición particular con el formato de la asignatura de Didáctica General, correspondiente a la formación de profesores en Facultad de Humanidades y Ciencias (FHUC-UNL). El equipo que conforma esta cátedra concibe la docencia universitaria basada en un trípode de enseñanza, investigación y extensión. A continuación haremos visibles modos concretos en que estas tres funciones se fueron articulando y retroalimentando.

\section{Cuando la práctica de enseñanza invita a investigar}

Como primer ejemplo mencionaremos una situación que dio lugar a los dos primeros proyectos de investigación a cargo de nuestro equipo, desarrollados en lo que actualmente ${ }^{4}$ es la FHUC. En lo personal, comienzo a trabajar allí a fines de la década del '80 en lo que aun era la cátedra de Conducción del Aprendizaje General. ${ }^{5}$ Las primeras percepciones que recogí estuvieron dadas por una desvalorización del saber pedagógico y didáctico respecto de los saberes disciplinares específicos y una gran desvinculación entre ambos ejes que organizaban la formación docente en el diseño curricular. Inmediatamente surgió la pregunta acerca del por qué de tal disociación entre saber pedagógico y saber disciplinar en una institución de formación docente y cómo era posible que lo específico — la docencia - no fuera valorada por la comunidad educativa. También me preguntaba cuáles podrían ser las instancias de confluencia y/o integración.

Esta situación generó luego dos proyectos de investigación casi simultáneos. Por un lado -y esto fue lo bueno de una extranjeridad que tenía respecto de esta institución que no conocía一, me llevó a indagar en la conformación de la propia institución, los proyectos que en ella se albergaron como así también en las visiones que docentes y egresados habían construido en torno al saber pedagógico- didáctico. Y un tramo especial, implicó analizar los enfoques a través de los cuales se había enseñado en esa cátedra. Esto llevó a comprender la complejidad del asunto, en la que se conjugaban muchas dimensiones y "actores" pero que tenían la particularidad de que casi nadie se visualizaba como parte del problema (Baraldi, 1996).

La otra inquietud, acerca de cómo lograr instancias de confluencia entre dos ejes de formación, dio lugar a un proyecto de investigación colectivo al que denominamos la "Integración de la Didáctica General con las Didácticas Específicas" ${ }^{6}$ y para el cual incorporamos rasgos de investigación-acción. Este proyecto posibilitó una experiencia inédita en el país, organizada a través de una cátedra colegiada entre profesoras de ciencias de la educación y profesoras formadas en las cinco disciplinas que al momento del proyecto tenían formación de profesores en la Facultad: Geografía, Historia, Matemática, Letras y Biología. Habíamos podido identificar — siguiendo una idea planteada por Elsie Rockwell- preguntas y ejes problemáticos inherentes a toda práctica de enseñanza cuyas respuestas eran abordadas desde las particularidades de cada disciplina. Encontramos un modo en que los estudiantes se formasen en diálogo con otros campos de conocimiento. Para llegar a esa etapa fueron necesarios momentos y trabajos específicos y un dispositivo de trabajo que permitía la constante revisión y modificación no sólo de nuestras prácticas sino de nuestros entendimientos (Baraldi, 1998) La conformación de ese equipo de trabajo también hizo factible en ese entonces el desarrollo de un Proyecto de Extensión de Cátedra enfocado a los procesos de comprensión en el cual trabajamos con tres escuelas públicas de la ciudad de Santa Fe (Baraldi, 2004). Este proyecto ya introdujo una dimensión muy importante en el modo de comprender la enseñanza y luego permitió la definición de otros trabajos.

\section{Cuando una situación educativa requiere un abordaje compartido surge un proyecto de extensión}

La sanción de la Ley de Educación Nacional 26206, junto a otras leyes y definiciones de las políticas educativas nacionales iniciadas en la primera década del siglo XXI, generó una serie de transformaciones y expectativas respecto de cambios para el sistema educativo. Por tener nuestros estudiantes, futuros profesores, fuerte inserción en la educación secundaria, hemos puesto —en los últimos años- especial atención al conocimiento de ese nivel educativo, procurando conocer las principales modificaciones esperadas para el mismo. Esto derivó en la definición de un proyecto de investigación a fin
4) Esta institución cambió cinco veces de nombre. Surge como Instituto del Profesorado, luego Instituto del Profesorado Básico, Escuela Universitaria, Facultad de Formación Docente, y actualmente Facultad de Humanidades y Ciencias. Para un mayor conoci- miento de estas transformaciones, cfr. Baraldi, 1996.

5) La denominación de Conducción del Aprendizaje estuvo vigente en el país en muchas instituciones de formación docente y de cuenta del exacerbado vínculo que se mantenía con la psico- logía. Si bien este vínculo se sostiene, no es la única disciplina que aporta a la comprensión de la enseñanza. Actualmente se hace hincapié en la relación ontológica que guarda la enseñanza respecto del aprendizaje y se plantea una clara distinción entre ambos procesos, dando centralidad al objeto de conocimiento de la didáctica, es decir, a la enseñanza 6) Subsidiado por la convocatoria CAI+D UNL del año 1993. 
de conocer procesos curriculares en la provincia de Santa Fe desarrollados a partir de la implementación de la ley mencionada. ${ }^{7}$ Pero también implicó el reconocimiento y la implicación en un conjunto de cuestiones que hacen a las prácticas docentes. En particular, haremos referencia a uno de sus desafíos, como fue la incorporación de la enseñanza de contenidos curriculares comunes (art. 88, 89, 92). Dichos contenidos aluden a un conjunto de temáticas que tienen que ser abordados en la escuela, tales como educación ambiental, derechos de los niños/as y adolescentes, diversidad cultural de los pueblos originarios, el ejercicio y construcción de la memoria colectiva, entre otros, y que tienen que ser enseñados independientemente del nivel o la modalidad. En todos los casos, por la complejidad de los temas, consideramos que su implementación demandaba un abordaje interdisciplinar. Pero, por sobre todo, requería un tratamiento interinstitucional, es decir un trabajo conjunto entre docentes de escuelas secundarias y docentes universitarios, cada uno aportando sus saberes y experiencias. En algunos casos saberes asociados a la apropiación de conocimientos sistemáticamente formulados, en otros a un saber experiencial de la propia formación docente. Y que eran (y son necesarios) proyectos interinstitucionales para potenciar y articular esa diversidad de saberes.

Entonces, sobre la base de comprender a la extensión como la posibilidad de abordar problemáticas en forma conjunta entre la Universidad y otras instituciones y grupos sociales, entendimos que los desafíos de la implementación de la Ley 26206 no competen solo a quienes están abocados a la enseñanza de un nivel, sino a la docencia en general y, en particular, a quienes estamos abocados a la formación de docentes. Desde este principio, y ante lo relevante del desafío de la incorporación de ciertas temáticas en la enseñanza del nivel, elaboramos a implementamos el Proyecto de Extensión de Interés Institucional denominado "Enseñar contenidos curriculares comunes: un desafío para la universidad y las escuelas secundarias". ${ }^{8}$ Dicho proyecto implicó distintas etapas de trabajo: recopilación de información específica en torno al aprendizaje de estas temáticas por parte de los estudiantes de la educación secundaria, la definición entre todos los docentes de un eje de trabajo vertebrador, ${ }^{9}$ procesos de formación de los profesores de ambas instituciones, ${ }^{10}$ hasta llegar a la definición de propuestas que, con distintos formatos, pudiesen sensibilizar a los estudiantes de la escuela secundaria respecto de las temáticas anteriormente enunciadas. Fue entonces cómo, a través de dramatizaciones, breves exposiciones, juegos con distintas reglas y propósitos, infografías, videos elaborados por los estudiantes se trabajó en torno a: movimientos sociales latinoamericanos, pueblos originarios, espacios públicos, consumo y contaminación, desigualdad social, entre otros temas, docentes y estudiantes, convocados durante una jornada institucional, se abocaron a su tratamiento. Quedaron en las escuelas materiales para continuar profundizando estos temas; y además de los informes correspondientes a la UNL y presentaciones a congresos ${ }^{11}$ elaboramos un video ${ }^{12}$ para documentar la experiencia.

El trabajo realizado implicó una excelente experiencia de formación de los estudiantes, en tanto pudieron pensar la enseñanza "en situación". De este modo su aproximación a distintas teorías quedó referenciada e interpelada por la experiencia, y la experiencia a su vez generó interrogantes para continuar estudiando.

En este proyecto, los estudiantes tuvieron un acercamiento directo a los jóvenes que habitan hoy las escuelas secundarias, a conocer sus expresiones, dificultades, posibilidades, demandas y deseos, como también a vivenciar las dinámicas específicas de este nivel educativo, con sus tiempos, sus códigos y sus prácticas. Alli pudieron visualizar las múltiples dimensiones que hacen a la complejidad de la práctica de enseñanza y conocer condiciones simbólicas y materiales en las que ésta tiene lugar.

Los docentes de las escuelas secundarias valoraron positivamente esta experiencia, manifestaron que nuestra presencia les había permitido organizar y desarrollar acciones que, a pesar de haber sido identificadas como necesarias, se tornaban difíciles de concretar. Igualmente valoraron que la Universidad no concurriera a observar y juzgar sino a conocer y trabajar conjuntamente.

El vínculo establecido con las escuelas secundarias hoy hace posible ofrecer nuevas instancias de formación a los estudiantes de la cátedra de Didáctica General de la UNL, en donde sus trayectos de formación están vinculados a proyectos institucionales de escuelas secundarias de la ciudad de Santa Fe. Esto exige por parte de ellos no solo estudiar sino participar de instancias colectivas de trabajo docente, lo cual hace que vayan construyendo una identidad con respecto a su futuro campo laboral.
7) Proyecto de Investigación, convocatoria CAID+D 2011, UNL: "Procesos de construcción curricular en la educación secundaria santafesina a partir de la Ley Nacional de Educación". Directora: Victoria Baraldi

8) El proyecto se aprobó por Res. "CS" $166 / 12$ y se implementó entre los años 2013-2015.
9) En este proyecto el eje organizador fue: "Identidad y ciudadanía en el contexto latinoamericano". 10) Lo cual implicó el análisis de bibliografía específica y la concreción de un Seminario Taller, con la invitación de Silvia Finocchio, quien no solo abordó definiciones conceptuales sino que compartió ideas en torno a estrategias específicas para el tratamiento del eje de trabajo definido conjuntamente. 11) Presentación en las Jornadas de Extensión del Mercosur organizadas por la Universidad Nacional de Centro (Tandil) y la Universidad de Passo Fundo (Brasil) (10 y 11 de abril de 2014) y presentación en Congreso Internacional de Extensión Universita- ria Universidad Nacional de Rosario (16 al 19 de septiembre de 2014). 12) El video puede consultarse en: http://www.fhuc.unl.edu.ar/pages/extension/programas-y-proyectos/proyectos-de-extension/educacion.php 


\section{G6 \\ en cuanto a los procesos, entendemos \\ que una cátedra que hace investigación \\ se asienta sobre la base de la \\ provisionalidad del conocimiento}

\section{La investigación y la extensión enriquecen las prácticas de enseñanza}

Demás está decir que tanto las prácticas de extensión como de investigación nutrieron, a lo largo de estos años, las prácticas de enseñanza. Estos aportes se pueden reconocer con relación a los procesos y a las producciones específicas.

En cuanto a los procesos, entendemos que una cátedra que hace investigación se asienta sobre la base de la provisionalidad del conocimiento. Esto tiene derivaciones en los procesos de transmisión, en tanto lejos quedan los dogmas y los estereotipos y se da lugar al reconocimiento del carácter polémico y controvertido de las humanidades y las ciencias sociales. Como dijera Stenhouse, enseñar un conocimiento es enseñar la naturaleza de ese conocimiento. En este caso, el conocimiento sobre la enseñanza siempre está atravesado por dimensiones teóricas, históricas y políticas. Asimismo, el trabajo colaborativo con instituciones educativas, en donde se ponen en juego perspectivas, saberes y voluntades, enriquece los procesos de formación de estudiantes y docentes. El enfrentarse a situaciones concretas de enseñanza, intervenir y decidir en torno a ellas, ha permitido el desarrollo de una praxis y al cultivo de la phronesis. Esto da un plus a la formación y enriquece notablemente los procesos de estudio.

En el marco de estas instancias, también quisimos desarticular un modo "cristalizado" de las instituciones de formación docente, en el que los estudiantes tratan de situarse de una manera "imperceptible" para observar y luego interpretar o hacer "coincidir" lo que observan con la o las teoría/s que están estudiando. Hoy el vínculo que pretendemos consolidar se basa en una relación de colaboración, de diálogo, de definición de proyectos conjuntos, sobre la base de que todos (docentes y estudiantes universitarios y docentes de educación secundaria) contamos con saberes construidos en distintas circunstancias y que, puestos en situación, permiten definiciones novedosas para enseñar. Estos vínculos han dado lugar a modos de intervención menos formales y más creativos por parte de los estudiantes universitarios. Lejos de centrarnos en una rigurosa planificación y su concreción en el aula, nos interesa que elaboren guiones de trabajo y tengan la suficiente ductilidad para transformarlos acorde al devenir de la propia práctica.

Pero además del tipo de procesos que se generan, es oportuno resaltar que distintas producciones luego se incluyen como materiales de enseñanza. De este modo, ponencias en congresos, artículos, libros, videos, fueron incorporados en las distintas versiones del programa de la cátedra. Los "principios y dimensiones de una perspectiva didácticas" (Baraldi, Bernik, Díaz, 2012), la indagación relativa a los "saberes del docente", los informes sobre los "cambios en las políticas educativas actuales", entre otras cuestiones, fueron y son parte de la bibliografía de Didáctica General. ${ }^{13}$

Estas producciones nos han permitido reformular el programa año tras año, el cual también se nutre de otros resultados de investigación en didáctica, de las inquietudes y procesos de formación del equipo y de los aprendizajes que, como docentes, vamos transitando al reflexionar sobre nuestra práctica docente.

13) Para mayores detalles consultar el programa de Didáctica General en: http://www.fhuc.unl.edu.ar/programas/ programas_aprobados.php 


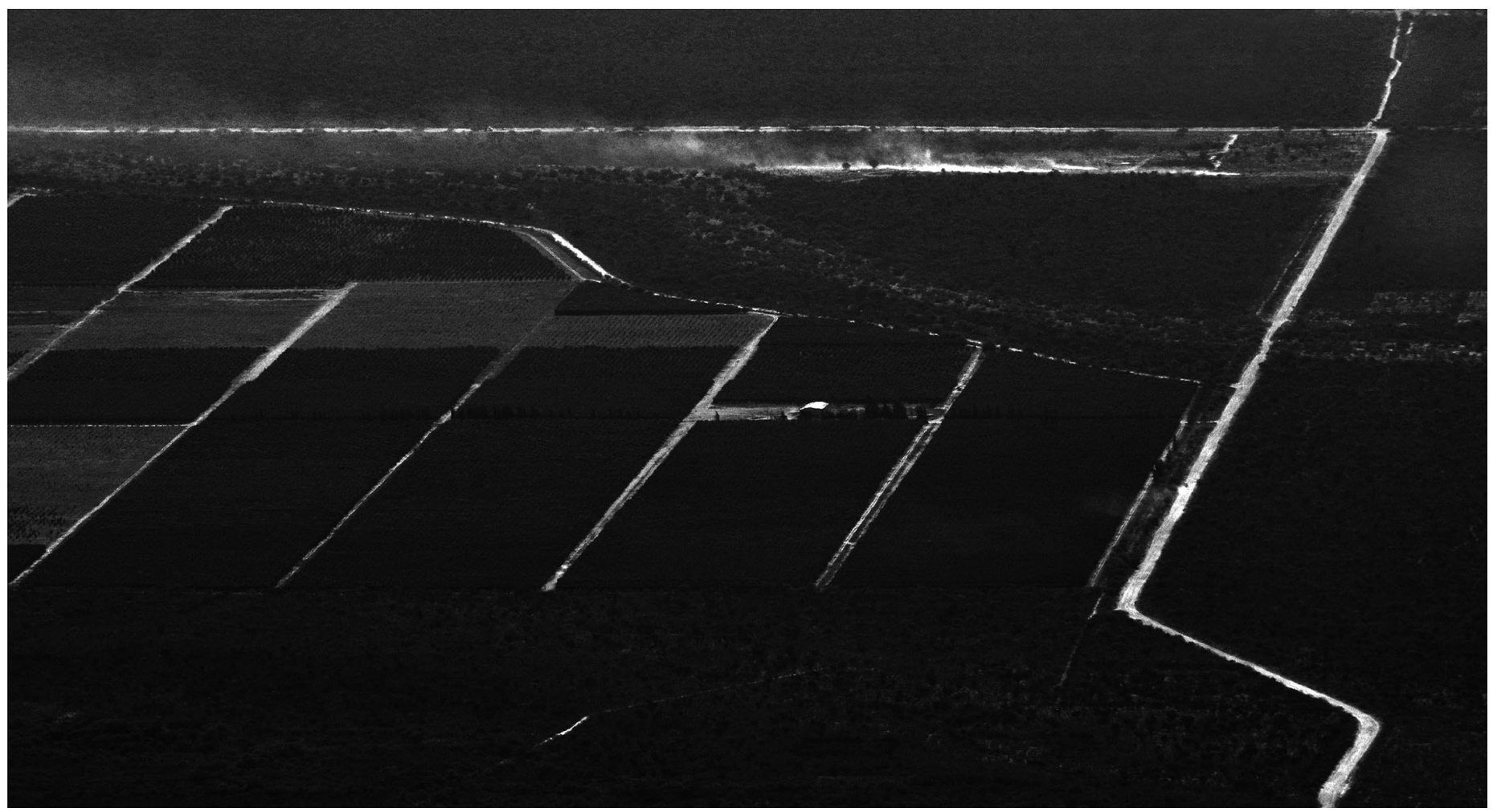

(C) Oscar Dechiara

\section{6}

el trabajo colaborativo con instituciones educativas, en donde se ponen en juego perspectivas, saberes y voluntades, enriquece los procesos de formación de estudiantes y docentes 


\section{6}

\author{
hemos enfatizado en la necesidad de trabajar \\ entre disciplinas y entre instituciones y le hemos \\ dado lugar un lugar protagónico a la voz de los \\ estudiantes, haciendo posible y visible una \\ dimensión estética en los procesos de formación
}

\section{Principios que sustentan el trabajo de la cátedra}

Para finalizar, así como al inicio del texto identificamos dos grandes coordenadas desde donde escribimos (la UNL y la didáctica) es justo mencionar que el trabajo realizado se sostiene sobre la base de supuestos y principios compartidos por el equipo de cátedra. En tal sentido, entendemos a la práctica docente como una relación entre sujetos mediada por el conocimiento y la finalidad de la enseñanza (Guyot y Giordano, 1997), lo cual implica la necesidad de una constante reflexión acerca de las prácticas de conocimiento. Las prácticas docentes necesitan interrogarse, entre otras cuestiones, en torno a la situacionalidad histórica, la relación saber/poder y los vínculos con otras disciplinas, sin olvidar que toda práctica docente tiene siempre una dimensión política que le otorga un sentido e implica la toma de decisiones.

En todos estos años hemos hecho énfasis en develar los dispositivos que regulan las prácticas educativas, y por ello hicimos hincapié en el análisis en profundidad de algunas de las dimensiones que las constituyen, como son la cuestión del tiempo (Baraldi, 2016) y los campos de conocimiento (Bernik, 2016).

Hemos enfatizado en la necesidad de trabajar entre disciplinas y entre instituciones (Baraldi, Bernik y Díaz, 2012) y le hemos dado lugar un lugar protagónico a la voz de los estudiantes, haciendo posible y visible una dimensión estética en los procesos de formación.

\section{Referencias bibliográficas}

Baraldi, V. (1996). El lugar de la didáctica en la formación docente. Historia de una problemática compleja. Santa Fe: Centro de Publicaciones de la Universidad Nacional del Litoral.

Baraldi, V. (1998) La didáctica en los 90. Radiografía de un proceso en construcción (Mimeo).

Baraldi, V. (2004). "Facultad- Escuela: la comprensión en el aula, un desafío para profesores y alumnos". En UNL Proyectos de extensión 1995-2001 (pp. 52-53). Santa Fe: UNL.

Baraldi, V. (2016). Tiempo y didáctica: itinerarios para pensar la enseñanza. Tesis de Doctorado en Educación. Universidad Nacional de Entre Ríos.

Baraldi, V.; Bernik, J. y Díaz, N. (2012). Una didáctica para la formación docente. Dimensiones y principios para la enseñanza. Santa Fe: Ediciones UNL.

Bernik, J. (2016). Campos de conocimiento y enseñanzas. Tesis de Doctorado en Educación. Universidad Nacional de Entre Ríos.

Camilloni, A. (1996). De herencias, deudas y legados. En Corrientes Didácticas contemporáneas. Buenos Aires: Paidós Educador.

Guyot, V. y Giordano, M. (1997). Los sujetos de la práctica docente como sujetos de conocimiento. Alternativas, 1(4), marzo. Laboratorio de Alternativas Educativas. Universidad Nacional de San Luis.

Universidad Nacional del Litoral (2010) Plan de desarrollo Institucional 2010-2019. Aprobado por Res. "A.U" 07/10. Santa Fe.

Universidad Nacional del Litoral (2012). Estatuto. Res "A.U" 04/2012. Santa Fe. 